ЗАКАЧКА ДИОКСИДА СЕРЫ В ПЛАСТ, ЧАСТИЧНО НАСЫЩЕННЫЙ ВОДОЙ

SULFUR DIOXIDE INJECTION INTO LAYER

PARTIALLY SATURATED WITH WATER

\author{
М.К. Хасанов
}

Башкирский государственный университет, филиал, г. Стерлитамак, Российская Федерация

\title{
М.В. Столповский
}

Уфимский государственный нефтяной технический университет, г. Уфа, Российская Федерация

Marat K. Khasanov

Bashkir State University, Branch, Sterlitamak, Russian Federation

MaksimV. Stolpovskii

Ufa State Petroleum Technological University, Ufa, Russian Federation e-mail: hasanovmk@mail.ru

Аннотация. Представлена математическая модель инжекции жидкой двуокиси серы в пласт, частично насыщенный водой, сопровождающейся образованием газового гидрата двуокиси серы на подвижной фронтальной поверхности, распространяющейся в глубь пласта. Получены распределения давления и температуры в пласте для различных значений его проницаемости. Исследованы зависимости координаты и температуры границы образования газогидрата диоксида серы от давления инжекции, 
проницаемости и начального давления пласта. Установлено, что при достаточно высоких значениях давления инжекции и проницаемости пласта, а также низких значениях начального давления пласта температура пористой среды на границе образования газогидрата диоксида серы может повышаться выше равновесной температуры разложения газогидрата диоксида серы. Это соответствует возникновению в пласте промежуточной зоны, насыщенной смесью воды, диоксида серы и его газогидрата, находящихся в состоянии фазового равновесия. Исследована зависимость предельного значения давления инжекции диоксида серы, выше которого возникает промежуточная зона, от проницаемости пласта и его начальных температуры и давления. Установлено, что с уменьшением проницаемости пласта и его начальной температуры величина предельного значения давления инжекции увеличивается. Поэтому промежуточная область, насыщенная смесью воды, диоксида серы и его газогидрата, находящихся в состоянии термодинамического равновесия, возникает при высоких значениях проницаемости пласта и его начальной температуры.

Abstract. A mathematical model of liquid sulfur dioxide injection into the layer, partially saturated with water, accompanied by the formation of sulfur dioxide gas hydrate on a moving frontal surface extending deep into the layer, is presented. The pressure and temperature distribution in the reservoir is obtained for different values of its permeability. The dependences of the coordinates and temperatures of the boundary of formation of sulfur dioxide gas hydrate on the injection pressure, permeability and initial formation pressure are investigated. It has been established that with sufficiently high values of injection pressure and reservoir permeability, as well as low values of the initial formation pressure, the temperature of the porous medium at the boundary of formation of sulfur dioxide gas hydrate can rise above the equilibrium decomposition temperature of sulfur dioxide gas hydrate. This corresponds to the formation in the reservoir of an intermediate zone saturated with a mixture of water, sulfur dioxide and its gas hydrate, which are in a state of phase equilibrium. The dependence of the 
limit value of the pressure of injection of sulfur dioxide, above which an intermediate zone occurs, on the permeability of the reservoir and its initial temperature and pressure is investigated. It has been established that with a decrease in the permeability of the reservoir and its initial temperature, the value of the limiting injection pressure increases. Therefore, an intermediate region saturated with a mixture of water, sulfur dioxide and its gas hydrate, which are in a state of thermodynamic equilibrium, occurs at high values of reservoir permeability and its initial temperature.

Ключевые слова: математическая модель; пористая среда; фильтрация; газогидраты; диоксид серы; метан; автомодельное решение

Key words: mathematical model; porous medium; filtration; gas hydrates; sulfur dioxide; methane; self-similar solution

\section{Введение}

Утилизация вырабатываемой промышленными объектами двуокиси серы в истощенных месторождениях газа является одним из способов снижения эмиссии диоксида серы в атмосферу [1-3]. Однако при длительном подземном хранении двуокиси серы в виде флюида существует риск ее утечки на поверхность. Риск утечки можно значительно уменьшить путем перевода двуокиси серы в твердое газогидратное состояние [4-7]. Математические модели процессов образования газогидрата в природном пласте приведены, например, в работах [8-10]. В данной работе представлена математическая модель закачки жидкой двуокиси серы в пласт, частично насыщенный водой, сопровождающейся образованием газового гидрата двуокиси серы. Исследованы условия, при которых возникает протяженная область образования газогидрата диоксида серы. 


\section{Постановка задачи}

На рисунке 1 приведена фазовая диаграмма системы « $\mathrm{SO}_{2}-\mathrm{H}_{2} \mathrm{O}$ », на которой представлены термобарические условия существования газогидрата $\mathrm{SO}_{2}$ [4]. На рисунке 1 кривая 1 соответствует трехфазному равновесию между газообразным диоксидом серы, его газогидратом и водой; кривая 2 - двухфазному равновесию между жидким и газообразным диоксидом серы; а кривая 3 - равновесию между жидкой двуокисью серы, газогидратом и водой. Газогидрат диоксида серы существует в области параметров, лежащей левее кривой 3 и выше кривой 1 . В верхней квадрупольной точке $Q_{2}\left(T_{Q}=285,1 \mathrm{~K}\right.$ и $p_{Q}=0,233$ МПа $)$ жидкий и газообразный диоксид серы, а также его газогидрат и вода находятся в равновесии.

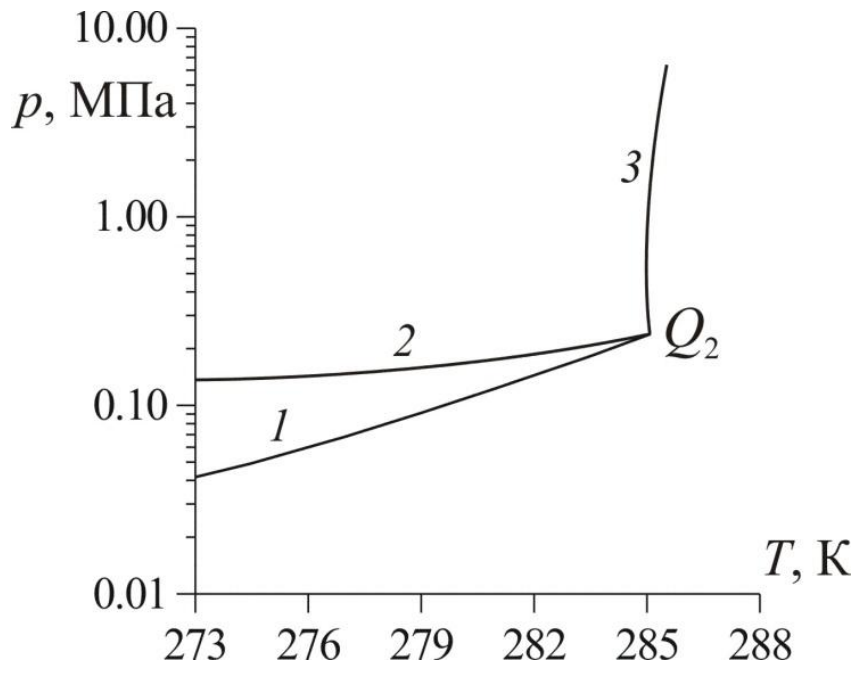

Рисунок 1. Фазовая диаграмма системы « $\mathrm{SO}_{2}-\mathrm{H}_{2} \mathrm{O}$ »

Рассмотрим полубесконечный пласт, который в начальный момент времени насыщен водой с исходной насыщенностью $S_{w 0}$ и метаном. Будем полагать, что в исходном состоянии температура пласта $T_{0}$ ниже температуры $T_{Q}$, соответствующей квадрупольной точке, а исходное давление $p_{0}$ соответствует термодинамическим условиям существования диоксида серы в жидком состоянии и его газогидрата (т.е. выше кривой 2 на рисунке 1). Следовательно, в рассматриваемом случае исходное 
состояние системы соответствует термобарическим условиям существования газогидрата $\mathrm{SO}_{2}$. Положим, что через левую границу пласта непрерывно нагнетается жидкая двуокись серы, давление $p_{e}$ и температура $T_{e}$ которой соответствуют условиям существования газогидрата $\mathrm{SO}_{2}$. При указанных термобарических условиях в результате контакта воды и двуокиси серы в пласте возникает область, насыщенная диоксидом серы и его газогидратом.

Начальные и граничные условия имеют вид:

$$
\begin{gathered}
t=0: \quad S_{w}=S_{w 0}, \quad T=T_{0}, \quad p=p_{0} \quad(x \geq 0) \\
x=0: \quad T=T_{e}, \quad p=p_{e} \quad(t>0) .
\end{gathered}
$$

В данной работе будем рассматривать модель с поршневым вытеснением метана жидкой двуокисью серы. Тогда можно полагать, что образование газогидрата $\mathrm{SO}_{2}$ происходит на фронтальной поверхности, совпадающей с поверхностью вытеснения метана двуокисью серы (рисунок 2).

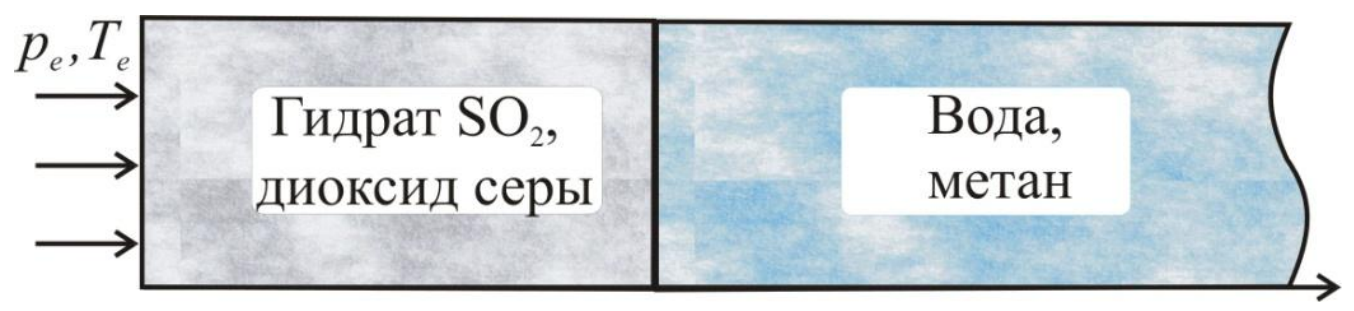

Рисунок 2. Схема задачи

\section{Основные уравнения}

Пусть величина начальной водонасыщенности пористого пласта не превышает 0,2, тогда воду можно считать неподвижной. В этом случае система основных уравнений в прямолинейно-параллельном случае имеет вид [8-10]:

$$
\frac{\partial}{\partial t}\left(\rho_{i} m S_{i}\right)+\frac{\partial}{\partial x}\left(\rho_{i} m S_{i} \cup_{i}\right)=0
$$




$$
\begin{gathered}
\rho c \frac{\partial T}{\partial t}+\rho_{i} c_{i} m S_{i} \cup_{i} \frac{\partial T}{\partial x}=\frac{\partial}{\partial x}\left(r \lambda \frac{\partial T}{\partial x}\right) ; \\
m S_{i} \cup_{i}=-\frac{k_{i}}{\mu_{i}} \frac{\partial p}{\partial x}, \quad k_{i}=k_{0} S_{i}^{3}(i=s, g) ; \\
\rho_{s}=\rho_{0 s} \exp \left(\beta_{s}\left(p-p_{0}\right)\right), \quad p=\rho_{g} R_{g} T .
\end{gathered}
$$

Здесь $x$ - координата; $t$ - время; $m$-пористость; $p$-давление; $T$-температура; нижние индексы $i=s, g$ относятся соответственно к параметрам диоксида серы и метана; $S_{i}$-насыщенность пор; $\rho_{i}-$ плотность; $v_{i}$ - действительная средняя скорость; $k_{0}$ - абсолютная проницаемость пористой среды; $k_{i}-$ фазовая проницаемость; $\beta_{s}$ - коэффициент сжимаемости жидкой двуокиси серы; $c_{i}-$ удельная теплоемкость; $\mu_{i}-$ динамическая вязкость; $R_{\mathrm{g}}$ - газовая постоянная метана; $\rho c$ и $\lambda$-эффективные значения объемной теплоемкости и коэффициента теплопроводности для насыщенного пласта.

На границе между зонами выполняются соотношения, вытекающие из условий баланса массы воды, двуокиси серы и метана, а также баланса теплоты:

$$
\begin{gathered}
-\frac{k_{s}}{\mu_{s}} \frac{\partial p_{1}}{\partial x}=m\left(\frac{\rho_{h}}{\rho_{s}} S_{h} G+S_{s}\right) V_{n}-\frac{k_{g}}{\mu_{g}} \frac{\partial p_{2}}{\partial x}=m S_{g} V_{n} \\
m S_{\mathrm{h}} \rho_{\mathrm{h}}(1-G) V_{n}=m S_{w 0} \rho_{w} V_{n}, \quad \lambda \frac{\partial T_{1}}{\partial x}-\lambda \frac{\partial T_{2}}{\partial x}=m \rho_{h} L_{h} S_{h} V_{n}
\end{gathered}
$$

Здесь $G$-относительная массовая концентрация диоксида серы в газогидрате; $S_{w 0}$-начальная водонасыщенность пласта; $L_{h}-$ удельная теплота образования газогидрата $\mathrm{SO}_{2}$ из жидкой двуокиси серы и воды; нижние индексы $j=1,2$ относятся соответственно к параметрам в первой и второй областях; $V_{n}$-скорость движения границы образования газогидрата диоксида серы. Здесь и далее нижний индекс $n$ относится к 
параметрам на поверхности между зонами, а нижние индексы $h$ и $w-$ соответственно к параметрам газогидрата и воды.

Из условия баланса массы воды (третьего соотношения системы (3)) для величины гидратонасыщенности в первой зоне имеем:

$$
S_{h}=\frac{\rho_{w} S_{w 0}}{\rho_{h}(1-G)}
$$

На основе (2) уравнения пьезопроводности и температуропроводности в каждой из зон пласта запишутся в виде:

$$
\begin{aligned}
& \frac{\partial p_{(1)}}{\partial t}=\chi_{(i)}^{(p)} \frac{\partial}{\partial x}\left(\frac{\partial p_{(1)}}{\partial x}\right), \frac{\partial T_{(1)}}{\partial t}=\chi^{(T)} \frac{\partial}{\partial x}\left(\frac{\partial T_{(1)}}{\partial x}\right)+\chi^{(T)} \mathrm{X}_{(1)} \frac{\partial p_{(1)}}{\partial x} \frac{\partial T_{(1)}}{\partial x} \\
& \frac{\partial p_{(2)}}{\partial t}=\chi_{(2)}^{(p)} \frac{\partial}{\partial x}\left(\frac{\partial p_{(2)}^{2}}{\partial x}\right), \quad \frac{\partial T_{(2)}}{\partial t}=\chi^{(T)} \frac{\partial}{\partial x}\left(\frac{\partial T_{(2)}}{\partial x}\right)+\chi^{(T)} \mathrm{X}_{(2)} \frac{\partial p_{(2)}^{2}}{\partial x} \frac{\partial T_{(2)}}{\partial x},
\end{aligned}
$$

где $\chi_{(1)}^{(p)}=\frac{k_{s}}{\mu_{s} m\left(1-S_{h}\right) \beta_{s}} ; \chi_{(2)}^{(p)}=\frac{k_{g} p_{0}}{\mu_{g} m\left(1-S_{w 0}\right)} \chi^{(T)}=\frac{\lambda}{\rho c}$;

$$
\mathrm{X}_{(1)}=\frac{\rho_{0 s} c_{s} k_{s}}{\lambda \mu_{s} \beta_{s}} ; \mathrm{X}_{(2)}=\frac{c_{g} k_{g}}{2 \lambda R_{g} T_{0} \mu_{g}}
$$

\section{Автомодельное решение}

Введем автомодельную переменную: $\xi=x / \sqrt{\chi^{(T)} t}$. Для автомодельной переменной на основе (4) и (5) получим решения для распределения давления и температуры в каждой из зон пласта:

$$
p_{(1)}=p_{(\mathrm{n})}+\frac{\left(p_{\mathrm{e}}-p_{(\mathrm{n})}\right) \int_{\xi}^{\xi_{(n)}} \exp \left(-\frac{\xi^{2}}{4 \eta_{(1)}}\right) d \xi}{\int_{0}^{\xi_{(n)}} \exp \left(-\frac{\xi^{2}}{4 \eta_{(1)}}\right) d \xi}, 0<\xi<\xi_{(n)}
$$




$$
\begin{gathered}
T_{(1)}=T_{(n)}+\frac{\left(T_{e}-T_{(n)}\right) \int_{\xi}^{\xi_{(n)}} \exp \left(-\frac{\xi^{2}}{4}-\mathrm{X}_{(1)} p_{(1)}\right) d \xi}{\int_{0}^{\xi_{(n)}} \exp \left(-\frac{\xi^{2}}{4}-\mathrm{X}_{(1)} p_{(1)}\right) d \xi}, 0<\xi<\xi_{(n)} ; \\
p_{(2)}^{2}=p_{0}^{2}+\frac{\left(p_{(n)}^{2}-p_{0}^{2}\right) \int_{\xi}^{\infty} \exp \left(-\frac{\xi^{2}}{4 \eta_{(2)}}\right) d \xi}{\int_{\xi_{(n)}}^{\infty} \exp \left(-\frac{\xi^{2}}{4 \eta_{(2)}}\right) d \xi}, \xi_{(n)}<\xi<\infty ; \\
T_{(2)}=T_{0}+\frac{\left(T_{(n)}-T_{0}\right) \int_{\xi}^{\infty} \exp \left(-\frac{\xi^{2}}{4}-\mathrm{X}_{(2)} p_{(2)}^{2}\right) d \xi}{\int_{\xi_{(n)}}^{\infty} \exp \left(-\frac{\xi^{2}}{4}-\mathrm{X}_{(2)} p_{(2)}^{2}\right) d \xi} . \xi_{(n)}<\xi<\infty,
\end{gathered}
$$

где $\eta_{(j)}=\chi_{(j)}^{(p)} / \chi^{(T)}(j=1,2)$.

На основе соотношений (3) с учетом (6) и (7) получим соотношения для определения параметров на поверхности гидратообразования:

$$
\begin{gathered}
\frac{\left(p_{\mathrm{e}}-p_{(\mathrm{n})}\right) \exp \left(-\frac{\xi_{(n)}^{2}}{4 \eta_{1}}\right)}{\int_{0}^{\xi_{(\mathrm{n})}} \exp \left(-\frac{\xi^{2}}{4 \eta_{1}}\right) d \xi}=A_{(1)} \xi_{(n)} ; \\
\frac{\left(p_{(\mathrm{n})}^{2}-p_{0}^{2}\right) \exp \left(-\frac{\xi_{(\mathrm{n})}^{2}}{4 \eta_{2}}\right)}{\left.\int_{\xi_{(\mathrm{n})}^{\infty} \exp \left(-\frac{\xi^{2}}{4 \eta_{2}}\right) d \xi}^{\infty}{ }^{\infty}\right)} A_{(2)} p_{(n)} \xi_{(n)} ; \\
\frac{\left(T_{(n)}-T_{e}\right) \exp \left(-\frac{\xi_{(n)}^{2}}{4}-X_{(1)} p_{(n)}\right)}{\xi_{(n)} \exp \left(-\frac{\xi^{2}}{4}-X_{(1)} p_{(1)}\right) d \xi}-\frac{\left(T_{0}-T_{(n)}\right) \exp \left(-\frac{\xi_{(n)}^{2}}{4}-X_{(2)} p_{(n)}^{2}\right)}{\int_{0}^{\infty} \exp \left(-\frac{\xi^{2}}{4}-X_{(2)} p_{(2)}^{2}\right) d \xi}=B \xi_{(n)},
\end{gathered}
$$

где $A_{(1)}=\frac{m \mu_{\mathrm{s}} \chi^{(T)}}{2 k_{s}}\left(\frac{\rho_{\mathrm{h}} G S_{\mathrm{h}}}{\rho_{0 \mathrm{~s}}}+1-S_{\mathrm{h}}\right) ; A_{(2)}=\frac{m \mu_{g} \chi^{(T)}}{k_{g}}\left(1-S_{w 0}\right) ; B=\frac{m \rho_{h} L_{h} S_{h}}{2 \rho c}$. 
В результате численного решения системы уравнений (8)-(10) определяются автомодельная координата поверхности гидратообразования $\xi_{(n)}$, а также значения давления и температуры на ней.

\section{Результаты расчетов}

На рисунке 3 приведены распределения давления и температуры в пласте при различных значениях абсолютной проницаемости пласта: $k_{0}=10^{-15} \mathrm{M}^{2}(a)$ и $k_{0}=10^{-14} \mathrm{M}^{2}(b)$. Штриховая линия - температура верхней квадрупольной точки. Для остальных параметров приняты следующие значения: $\quad m=0,2 ; \quad S_{w 0}=0,2 ; \quad p_{\mathrm{e}}=5 \mathrm{MПа} ; \quad p_{0}=4,5 \mathrm{MПа} ; \quad T_{0}=280 \mathrm{~K}$; $T_{e}=275 \mathrm{~K} ; G=0,372 ; \mu_{s}=3,68 \cdot 10^{-4} \Pi \mathrm{a} \cdot \mathrm{c} ; \mu_{g}=1 \cdot 10^{-4} \Pi \mathrm{a} \cdot \mathrm{c} ; \beta_{s}=1,35 \cdot 10^{-9} \Pi^{-1}$; $\lambda=2 \mathrm{BT} /(\mathrm{M} \cdot \mathrm{K}) ; \quad \rho c=2 \cdot 10^{6}$ Дж/(К·Кг); $\rho_{h}=1390 \kappa \Gamma / \mathrm{m}^{3} ; \quad \rho_{w}=1000 \kappa \Gamma / \mathrm{m}^{3} ;$ $\rho_{0 s}=1434$ кг $/ \mathrm{M}^{3} ; c_{s}=1350$ Дж/(К·кг); $L_{h}=2,62 \cdot 10^{5}$ Дж/кг.

Согласно рисунку 3, с повышением проницаемости пласта скорость движения поверхности гидратообразования возрастает. Это обусловлено тем, что движение данной поверхности лимитируется скоростью течения жидкого диоксида серы, которая согласно закону Дарси увеличивается с ростом проницаемости пласта.

Также рисунок 3 показывает, что с увеличением проницаемости пласта происходит рост температуры на поверхности фазового перехода. Это обусловлено тем, что образование газогидрата двуокиси серы сопровождается выделением скрытой теплоты фазовых переходов, а с повышением проницаемости происходит возрастание скорости поверхности фазовых переходов (т.е. интенсивности гидратообразования). Кроме того, поскольку часть тепла, выделяющегося на поверхности фазовых переходов, отводится через более холодную левую границу пласта, то с увеличением координаты поверхности гидратообразования происходит снижение интенсивности отвода тепла через левую границу пласта. 


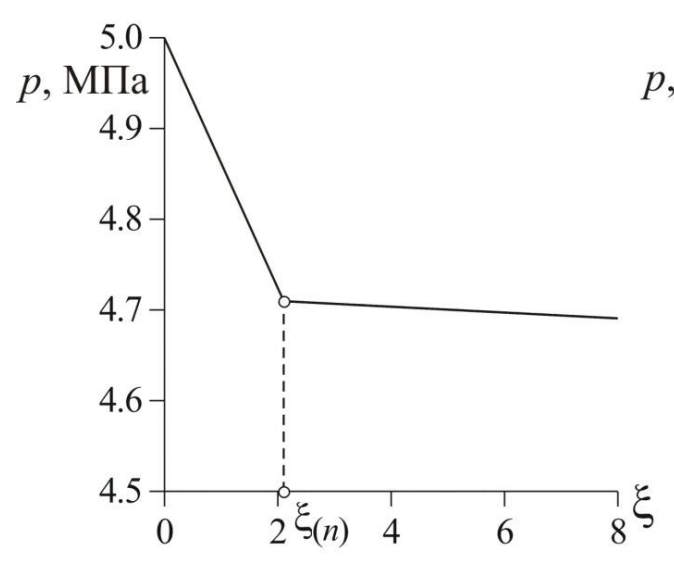

$b$

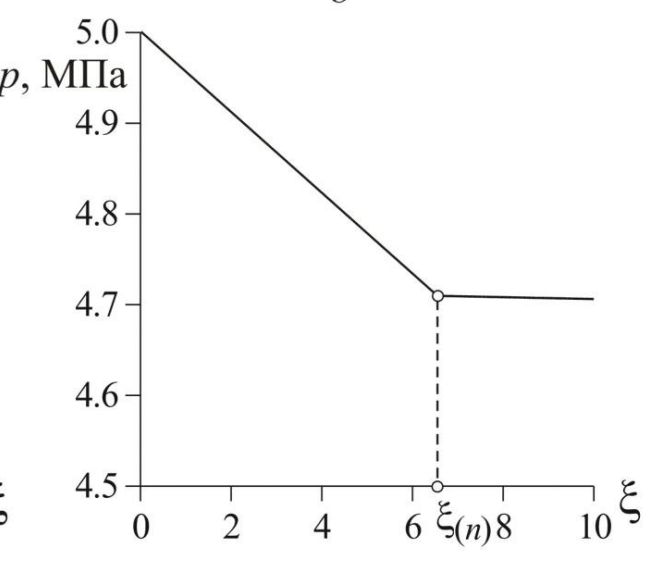

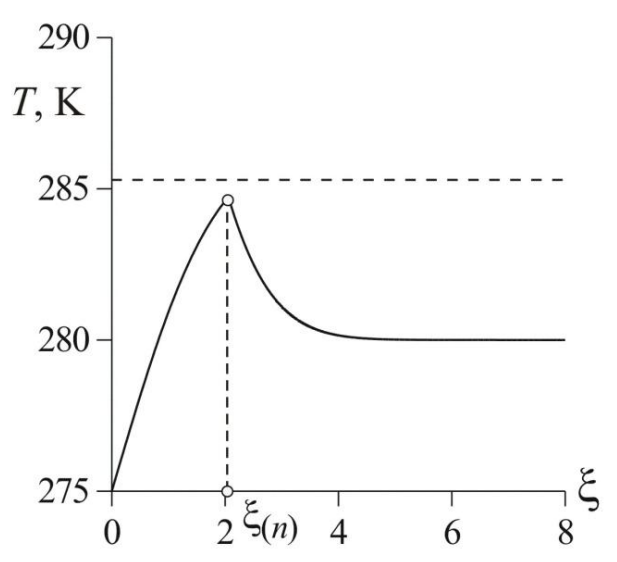

a)

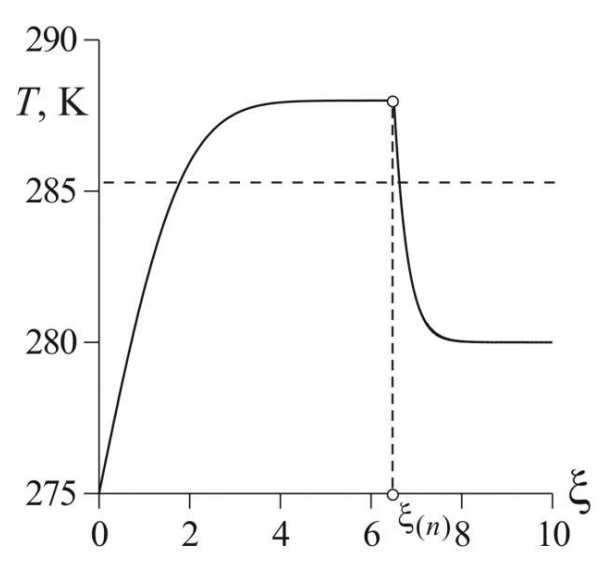

б)

Штриховая линия - температура верхней квадрупольной точки

Рисунок 3. Распределение давления и температуры в пласте при различных значениях абсолютной проницаемости пласта:

$$
k_{0}=10^{-15} \mathrm{M}^{2}\left(\text { a) и } k_{0}=10^{-14} \mathrm{M}^{2}(\text { б) }\right.
$$

Указанные факторы, согласно рисунку 3, приводят к росту температуры на данной границе при увеличении проницаемости пласта. Вследствие этого при достаточно больших значениях проницаемости пласта температура на границе гидратообразования может превысить равновесную температуру разложения газогидрата диоксида серы (штриховая линия), что будет соответствовать возникновению промежуточной области, насыщенной смесью воды, диоксида серы и его газогидрата, находящихся в состоянии термодинамического равновесия. Так, согласно рисунку 3, при низком значении проницаемости пласта (случай $a$ ) температура на границе образования газогидрата диоксида серы ниже, чем температура верхней квадрупольной точки. Следовательно, в этом случае модель с фронтальной границей образования газогидрата 
диоксида серы дает адекватное математическое описание процесса. При более высоких значениях проницаемости пласта (случай б) температура на границе образования газогидрата диоксида серы поднимается выше температуры верхней квадрупольной точки. В этом случае для адекватного описания процесса необходимо использовать модель с протяженной областью фазовых переходов.

На рисунке 4 приведена зависимость координаты и температуры границы образования газогидрата от начального давления (a) при $p_{\mathrm{e}}=4,5 \mathrm{MПа} \mathrm{и} \mathrm{от} \mathrm{давления} \mathrm{инжекции} \mathrm{(б)} \mathrm{при} p_{0}=4,5$ МПа. Штриховая линия - температура квадрупольной точки. Абсолютная проницаемости пласта равна $10^{-14} \mathrm{M}^{2}$ (кривая 1) и $10^{-13} \mathrm{M}^{2}$ (кривая 2). Для остальных параметров приняты значения, соответствующие рисунку 3.
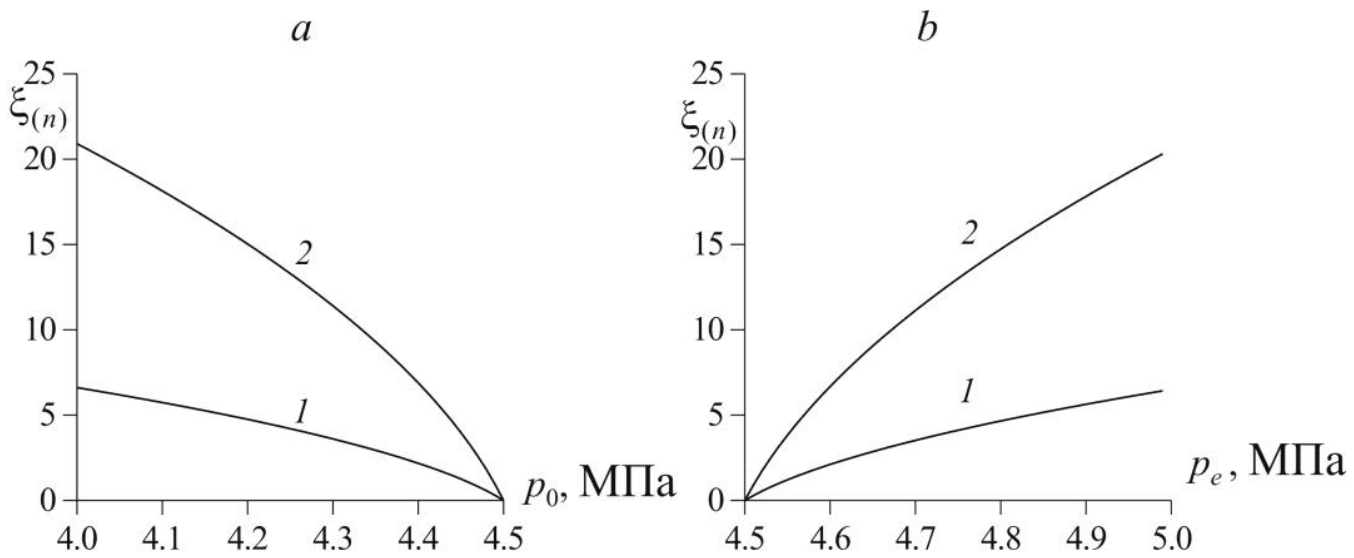

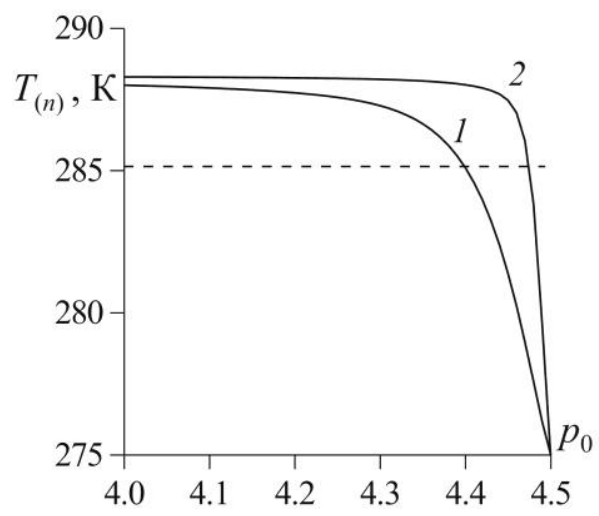

a)

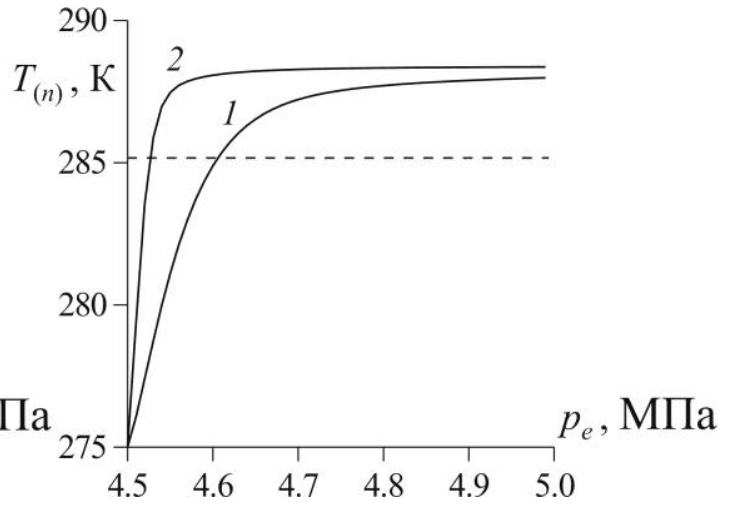

б)

Штриховая линия - температура квадрупольной точки; кривая $1-k_{0}=10^{-14} \mathrm{M}^{2} ;$ кривая $2-k_{0}=10^{-13} \mathrm{M}^{2}$

Рисунок 4. Зависимость координаты и температуры границы образования газогидрата от начального давления $(a)$ при $p_{\mathrm{e}}=4,5 \mathrm{MПа}$ и от давления инжекции (б) при $p_{0}=4,5 \mathrm{MПа}$ 
Согласно рисунку 4, с повышением давления инжекции и проницаемости, а также с уменьшением начального давления скорость движения поверхности гидратообразования возрастает. Это обусловлено тем, что движение данной поверхности лимитируется скоростью течения жидкого диоксида серы, которая согласно закону Дарси увеличивается с увеличением перепада давления в пласте.

Также рисунок 4 показывает, что с увеличением давления инжекции и проницаемости, а также с уменьшением начального давления происходит рост температуры на поверхности фазового перехода. Это обусловлено тем, что образование газогидрата двуокиси серы сопровождается выделением скрытой теплоты фазовых переходов, а с повышением давления инжекции и проницаемости, а также с уменьшением начального давления происходит возрастание скорости поверхности фазовых переходов (т.е. интенсивности гидратообразования). Кроме того, поскольку часть тепла, выделяющегося на поверхности фазовых переходов, отводится через более холодную левую границу пласта, то с увеличением координаты поверхности гидратообразования происходит снижение интенсивности отвода тепла через левую границу пласта. Указанные факторы, согласно рисунку 3, приводят к росту температуры на этой границе при увеличении давления инжекции проницаемости, а также при уменьшении начального давления.

Вследствие этого при достаточно больших значениях давления инжекции и проницаемости или малых значениях начального давления температура на границе гидратообразования может превысить равновесную температуру разложения газогидрата диоксида серы (штриховая линия), что будет соответствовать возникновению промежуточной области, насыщенной смесью воды, диоксида серы и его газогидрата, находящихся в состоянии фазового равновесия.

Были проведены расчеты для нахождения величины предельного значения давления инжекции диоксида серы $p_{\mathrm{pr}}$, выше которого возникает 
промежуточная зона, насыщенная смесью воды, диоксида серы и его газогидрата, находящихся в состоянии термодинамического равновесия.

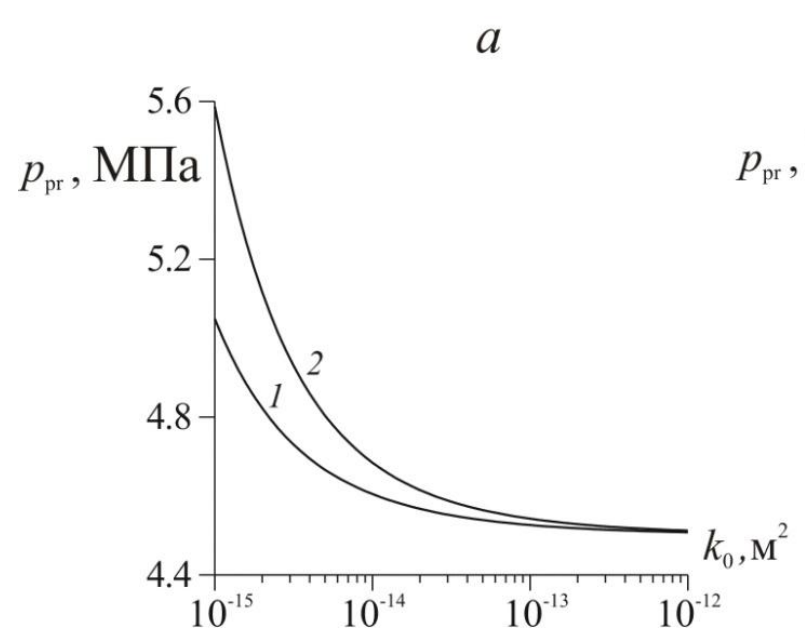

a) $b$

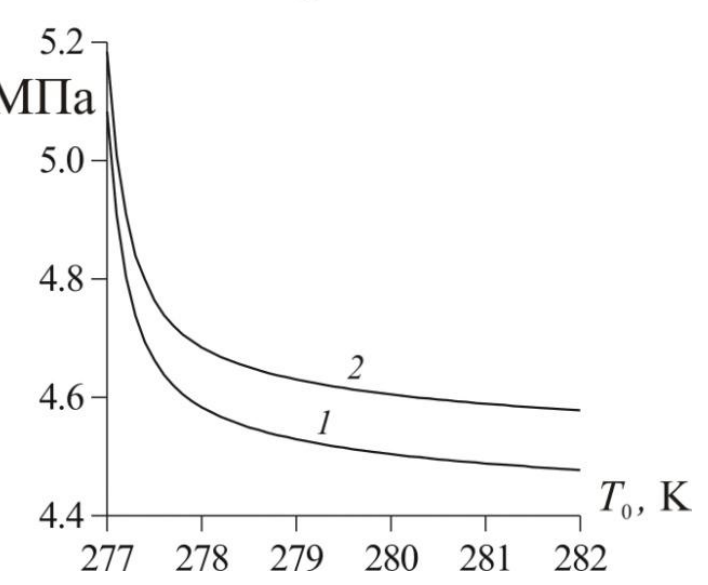

б)

Рисунок 5. Зависимость предельного значения давления от абсолютной проницаемости $(a)$ при $p_{0}=4,5 \mathrm{MПа,} T_{0}=280 \mathrm{~K}$ (кривая 1$)$ и $T_{0}=278 \mathrm{~K}$

(кривая 2) и от начальной температуры пласта (б) при $k_{0}=10^{-14} \mathrm{M}^{2}$, $p_{0}=4,4 \mathrm{MПа} \mathrm{(кривая} \mathrm{1)} \mathrm{и} p_{0}=4,5 \mathrm{MПа} \mathrm{(кривая} \mathrm{2)}$

На рисунке 5 приведена зависимость предельного значения давления от абсолютной проницаемости пласта (a) при $p_{0}=4,5 \mathrm{MПа}, T_{0}=280 \mathrm{~K}$ (кривая 1) и $T_{0}=278 \mathrm{~K}$ (кривая 2) и от начальной температуры пласта (б) при $k_{0}=10^{-14} \mathrm{M}^{2}, p_{0}=4,4 \mathrm{MПа} \mathrm{(кривая} \mathrm{1)} \mathrm{и} p_{0}=4,5$ МПа (кривая 2). Для остальных параметров приняты значения, соответствующие рисунку 3. Согласно рисунку 5, с уменьшением абсолютной проницаемости пласта и его начальной температуры, а также с увеличением начального давления величина предельного значения давления инжекции увеличивается. Это обусловлено тем, что с уменьшением абсолютной проницаемости пласта и его начальной температуры, а также с увеличением начального давления снижается температура на поверхности образования газогидрата диоксида серы. Поэтому для того чтобы температура на этой поверхности превысила равновесную температуру разложения газогидрата, необходимы низкая интенсивность отвода тепла через левую границу пласта и высокая интенсивность выделения скрытой теплоты гидратообразования, что 
реализуется при больших значениях давления инжекции и соответственно скорости движения границы фазовых переходов. Таким образом, промежуточная область, насыщенная смесью воды, диоксида серы и его газогидрата, находящихся в состоянии термодинамического равновесия, возникает при высоких значениях давления инжекции, проницаемости пласта и его начальной температуры.

\section{Вывод}

Построена математическая модель образования газового гидрата диоксида серы при инжекции жидкой двуокиси серы в пласт, насыщенный водой и метаном, Установлено, что с повышением проницаемости пласта и давления инжекции, а также с уменьшением начального давления пласта происходит увеличение температуры границы образования газогидрата диоксида серы. Показано, что при высоких значениях давления инжекции, начальной температуры пласта и его проницаемости пласта, а также низких значениях начального давления образование газогидрата $\mathrm{SO}_{2}$ будет происходить в протяженной области.

Исследование выполнено при финансовой поддержке РФФИ в рамках научного проекта № 19-08-00967.

\section{Список используемых источников}

1. Аншиц А.Г., Кирик Н.П., Верещагин С.Н. Экологическая и инженерная геология: подземное захоронение как возможность полной утилизации сернистых газов // Инженерная экология. 2009. № 3. С. 3-19.

2. Anshits A., Kirik N., Shibistov B. Possibilities of $\mathrm{SO}_{2}$ Storage in Geological Strata of Permafrost Terrain // Advances in the Geological Storage of Carbon Dioxide. 2006. Vol. 65. P. 93-102. DOI: 10.1007/1-4020-4471-2_09. 
3. Qi Li, Xiaochun Li, Ning Wei, Zhiming Fang. Possibilities and Potentials of Geological Co-Storage $\mathrm{CO}_{2}$ and $\mathrm{SO}_{2}$ in China // Energy Proccedia. 2011. Vol. 4. P. 6015-6020.

4. Истомин В.А., Якушев В.С. Газовые гидраты в природных условиях. М.: Недра, 1992. 236 с.

5. Дучков А.Д., Соколова Л.С., Аюнов Д.Е., Пермяков М.Е. Оценка возможности захоронения углекислого газа в криолитозоне Западной Сибири // Криосфера Земли. 2009. Т. 13. № 4. С. 62-68.

6. Чувилин Е.M., Гурьева О.М. Экспериментальное изучение образования гидратов $\mathrm{CO}_{2}$ в поровом пространстве промерзающих и мерзлых пород // Криосфера Земли. 2009. Т. 13. № 3. С. 70-79.

7. Chuvilin E.M., Guryeva O.M. The Role of Hydrate Formation Processes in Industrial $\mathrm{CO}_{2}$ Sequestration in Permafrost Area // Petroleum Abstracts. 2015. Vol. 55. No. 48. P. 96.

8. Shagapov V.Sh., Musakaev N.G., Khasanov M.K. Formation of Gas Hydrates in a Porous Medium during an Injection of Cold Gas // International Journal of Heat and Mass Transfer. 2015. Vol. 84. P. 1030-1039.

9. Khasanov M.K., Stolpovsky M.V., Gimaltdinov I.K. Mathematical Model for Carbon Dioxide Injection into Porous Medium Saturated with Methane and Gas Hydrate // International Journal of Heat and Mass Transfer. 2018. Vol. 127. P. 21-28.

10. Khasanov M.K., Stolpovsky M.V., Gimaltdinov I.K. Mathematical Model of Injection of Liquid Carbon Dioxide in a Reservoir Saturated with Methane and its Hydrate // International Journal of Heat and Mass Transfer. 2019. Vol. 132. P. 529-538. 


\section{References}

1. Anshits A.G., Kirik N.P., Vereshchagin S.N. Ekologicheskaya i inzhenernaya geologiya: podzemnoe zakhoronenie kak vozmozhnost' polnoi utilizatsii sernistykh gazov [Environmental and Engineering Geology: Underground Burial as a Possibility of Full Utilization of Sulfur Dioxide]. Inzhenernaya ekologiya - Engineering Ecology, 2009, No. 3, pp. 3-19. [in Russian].

2. Anshits A., Kirik N., Shibistov B. Possibilities of SO2 Storage in Geological Strata of Permafrost Terrain. Advances in the Geological Storage of Carbon Dioxide, 2006, Vol. 65, pp. 93-102. DOI: 10.1007/1-4020-4471-2_09.

3. Qi Li, Xiaochun Li, Ning Wei, Zhiming Fang. Possibilities and Potentials of Geological Co-Storage CO2 and SO2 in China. Energy Procedia, 2011, Vol. 4, pp. 6015-6020.

4. Istomin V.A., Yakushev V.S. Gazovye gidraty v prirodnykh usloviyakh [Natural Gas Hydrates]. Moscow, Nedra Publ., 1992. 236 p. [in Russian].

5. Duchkov A.D., Sokolova L.S., Ayunov D.E., Permyakov M.E. Otsenka vozmozhnosti zakhoroneniya uglekislogo gaza v kriolitozone Zapadnoi Sibiri [Assesment of Potential of West Siberian Permafrost for the Carbon Dioxide Storage]. Kriosfera Zemli - Earth's Cryosphere, 2009, Vol. 13, No. 4, pp. 62-68. [in Russian].

6. Chuvilin E.M., Gur'eva O.M. Eksperimental'noe izuchenie obrazovaniya gidratov $\mathrm{SO} 2 \mathrm{v}$ porovom prostranstve promerzayushchikh i merzlykh porod [Experimental Investigation of $\mathrm{CO} 2$ Gas Hydrate Formation in Porous Media of Frozen and Freezing Sediments]. Kriosfera Zemli - Earth's Cryosphere, 2009, Vol. 13, No. 3, pp. 70-79. [in Russian].

7. Chuvilin E.M., Guryeva O.M. The Role of Hydrate Formation Processes in Industrial CO2 Sequestration in Permafrost Area. Petroleum Abstracts, 2015, Vol. 55, No. 48, pp. 96. 
8. Shagapov V.Sh., Musakaev N.G., Khasanov M.K. Formation of Gas Hydrates in a Porous Medium during an Injection of Cold Gas. International Journal of Heat and Mass Transfer, 2015, Vol. 84, pp. 1030-1039.

9. Khasanov M.K., Stolpovsky M.V., Gimaltdinov I.K. Mathematical Model for Carbon Dioxide Injection into Porous Medium Saturated with Methane and Gas Hydrate. International Journal of Heat and Mass Transfer, 2018, Vol. 127, pp. 21-28.

10. Khasanov, M.K., Stolpovsky, M.V., Gimaltdinov, I.K. Mathematical Model of Injection of Liquid Carbon Dioxide in a Reservoir Saturated with Methane and its Hydrate. International Journal of Heat and Mass Transfer, 2019, Vol. 132, pp. 529-538.

\section{Сведения об авторах}

\section{About the Authors}

Хасанов Марат Камилович, канд. физ.-мат. наук, зав. кафедрой прикладной информатики и программирования, БашГУ, филиал, г. Стерлитамак, Российская Федерация

Marat K. Khasanov, Candidate of Physical and Mathematical Sciences, Head of Applied Informatics and Programming Department, BashSU, Branch, Sterlitamak, Russian Federation

e-mail: hasanovmk@mail.ru

Столповский Максим Владимирович, канд. физ.-мат. наук, доцент кафедры физики, УГНТУ, г. Уфа, Российская Федерация

Maksim V. Stolpovskii, Candidate of Physical and Mathematical Sciences, Assistant Professor of Physics Department, USPTU, Ufa, Russian Federation e-mail: s_maxim.pmm@mail.ru 